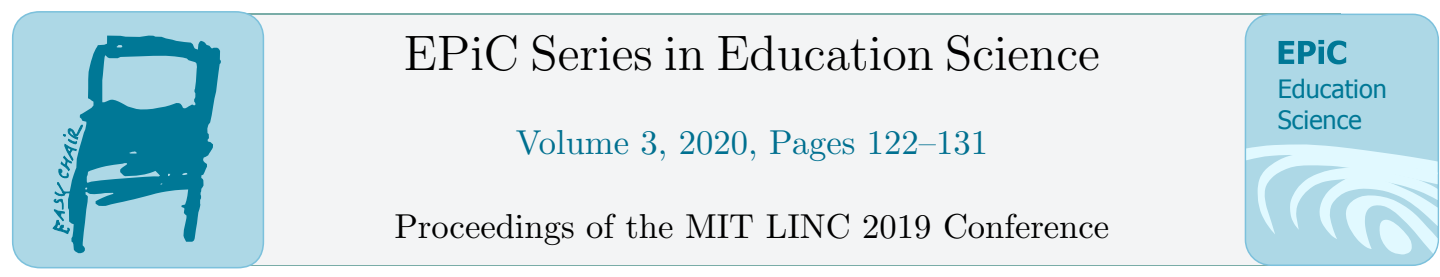

\title{
Simple Answers to Difficult Issues about Learning and Learners Internationally at pK-12 Levels
}

\author{
Ildiko Gyori \\ Humanmenu@gmail.com
}

A case study of novel ideas in learning and education with international relevance and local applicability

\section{Abstract}

The traditional educational model was designed in the industrial age of the 20th century. That model is outdated for various reasons. This paper is introducing a case study of a project, "The S-Cool Days Program," which applies new routines to reframe those aspects. The introduction of the Program does not require significant changes in the curriculum, can be realized in Art and Music classes, or as a homeroom activity in advisory class.

The Program was implemented in Europe, in two countries, in 3 different school types, one American School, a School Start-up and a traditional public school. The activities are introduced by creative art and craft sessions, are engaging enough to invite students to present their passion projects in the classroom, to take the first steps towards purpose-driven learning, learn how to focus on monitoring and expressing of emotions, among other things.

The goal is to have some school habits reframed, that results in mindset change. New values are introduced and through them, the class is transformed into a comfort zone for students to envision and follow their dreams. The expected changes can be realized by easy-to-do new routines, which maintain the newly introduced behaviors. The Program supports the 4C's; the 21st-century skills education is supposed to focus on: the creativity, communication, collaboration, and critical thinking. 
As visualized on social maps, the research realized before and after the Project shows changes in the social relations of the participating classes after the new routines were introduced. New mutual common choices were emerging in all participating grades, classes. The paper shares insights from participating teachers, how they felt in and after the Program, what changes did they identify in the classroom.

\section{Personal Motivation}

I am a psychologist neither a scientist, nor a researcher, but a single mother, who dedicated her free time and resources in the last seven years for finding and sharing simple answers to complicated issues present in the education system. The first week, my son entered the elementary education, we started to face many challenges, difficulties other parents, teacher, and kids meet as well. I was brave enough to find my way, allowed my son to develop in his rhythm, find his way to follow his passion. On the other hand, understanding and deeply believing in the concept of social responsibility, dedicated my time and resources to find solutions to the challenges - in a structured way so that more children, teachers, parents, schools, could benefit from it.

\section{Theoretical Background, "The Mind Age"}

Today, in the Mind Age, the traditional frameworks of economy and society are collapsing a global society is emerging surrounded by a global business environment and a technological development which has never been seen in the history of humanity before. As a result of these changes - and the technological tools and platforms behind- next to real life, virtual networks and "communities" are emerging at the social media platforms. Parallel with real life a teenager today may be "living" in virtual societies at the same time. For children, growing up in this transition time, it is not easy to find their way in school. Especially not, because the school system where they spend the majority of their day-time, and where they receive the majority of the feedback about themselves- is designed for the 20th century and served the aims and career paths of the Industrial Age. The context in which humans and children especially are supposed to navigate is getting more complex than ever before in the history of the earth. Schoolgirls and boys need a compass. Internet might be one compass, as ensures the free delivery of any know-how in seconds. Delivering wide range of know-how to schools on different platforms or channels might be one possible way to help them navigate in the school-system - yet delivers only a content-based solution, provides only answers to the question of "What shall we do and teach differently?" The answers to the content-based challenges of the 
education system (i.e., what to teach/learn at school with the help of which application) leave many concerns without answer which are present in the classroom - mainly "human factors," caused by the changes in the society.

We all know that to enable teachers and students in the education system to be efficient and successful, there is a need for re-defining the vision/aim of the entire school system itself. The only question is: How to do that? Do we need global standards for schools? How shall schools serve the society and the economy? Locally? At County or Country level? Do we need Global or Virtual schools?

These are essential questions, and a lot of us have been waiting for long for any decision to be made any change to be implemented. Decision makers are aware of the need for change, but there are so many complex issues involved that resolutions seem to be postponed. On the other hand, as a mother or psychologist I didn't have any week in the last several years without a parent calling me -literarycrying, and sharing a terrible story from the school of his/her school. I understood that there is no time to waste with waiting. Our children today at grade 1 or 6 have no years or decades for nation-wide agreement to emerge, a global learning platform to be introduced. They need solutions in the Aula now, which are easily applicable, instant and result in change welcomed by children, parents, teachers as well.

\section{How I started. The aim of the S-Cool Days Program}

It was clear that suggesting changes in the infrastructure and the technical toolkit is neither my competence nor my accountability. I decided to focus on the human part instead. Why? The outcomes articulated in 2003 by the Partnership for the 21st Century (P21) defined four skills for the 21st Century Education, known the $4 \mathrm{C}$ 's, the learning and innovation skills for the 21st Century to be acquired during school years. [1] "Communication, Critical Thinking, Collaboration and Creativity" are all human skills, which can be developed within the classroom. Another important factor raised my attention towards a need for a possible transformation of the in-classroom environment: the fact that the number of hours children spend at different types of screens is dramatically increasing, so does time spent at different Social Media Platforms, in virtual communities, sometimes far away from the reality. As expressed in the survey of Mediacom, in 2017 [2] "Teens rely heavily on social platforms to meet their needs such as Immediacy, Inspiration, Self-expression. The more criticism they experience, the more classmates are bullied from their eyes, the more they will escape to the consciously built up virtual reality.

The aim was clear to me: try to build up an attractive classroom culture, one in the "real life" which is "cool enough" to belong. At the same time, just like the virtual world, satisfies the needs for immediacy, inspiration, self-expression and becomes a platform for exercising the $4 \mathrm{C}$ 's, the relevant 21st Century skills. Making real life as interesting as the virtual world may be only "important" today, but will 
become a "must" soon, as generations are about to enter education who were nursed by tablets and other SMART tools from their birth. Children spend 8 hours at school and an average of six and a half hours a day in front of a screen. [2] Time spent within a human community has 1,5 hours of advantage, but if not used consciously, will probably melt. An alternative culture, a community should be built up in the real world which can offer the benefits of the screens and more. Schools have the chance to become an alternative, as they have much time to offer, classmates and teachers have attention to offer, but some routines and values should be changed to qualify for the race with the virtual world and communities. The name of the program refers to the direction of these changes - there is a need to transform the "I have to be here" feeling into the "I want to be here" feeling.

\section{What are the main characteristics of the S-Cool Days Program?}

The Program works without causing or demanding significant changes in the everyday curriculum can be delivered within the framework of Art, Music or Advisory. It is interesting, engaging and creative enough for children and teachers to engage them to participate. The set up offers enough attention to everyone, preferably with instant gratification. By introducing new routines, immediate mindset change occurs and remains in the Aula after the Program was finished. The fourth characteristic is the time-consciousness, quick and easy, preferably modulebased activities follow each other. At last but not at least, participation is fun, without shame, blame, guilt involved.

\section{Details of the work}

\subsection{Creativity and Communication}

From a very early age on children in modern societies spend the majority of their day-time in school, in a framework of 4-8 subjects, such as Mathematics Literature PE or Biology. In most cases their real passion is subject to after-school activities, resulting in that children do not know each-others free time activities, hobbies. A child is characterized by being on the "Principal list", or being a "C- in Math." The one, who is a passionate cook, has just a few opportunities at school to express the talent and receive attention and feedback from peers about his real interest.

To ensure, that all children receive attention at school, may express their creativity and are allowed to bring that into the group, the S-Cool Days Program starts with a module that invites kids to introduce their passion to classmates. The framework of the activity is a weekly routine that provides each child with the 
possibility to tell about his hobby or her preference. Everybody shares with the class at least three after-school or a weekend activity that he/she is proud of.

How do we realize it? They create a tree together, and hang on "flowers" or "leaves," introduce their passion, share the experience when and where they utilized, developed this passion. The "Passion Tree" is actualized every week, ensuring that "B+ Math Students" have the same weekly opportunity to be seen passionate, may demonstrate a keen interest and successful delivery of something important. The proper attention is given to each, regardless of the grades or the economic background of the family.

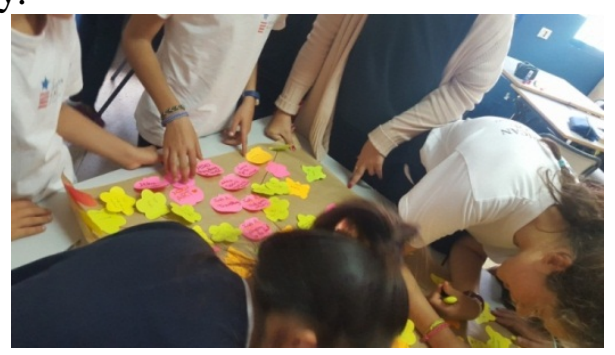

Image 1. Collaborating girls create the Passion Tree.

The American School of Las Palmas, 2018

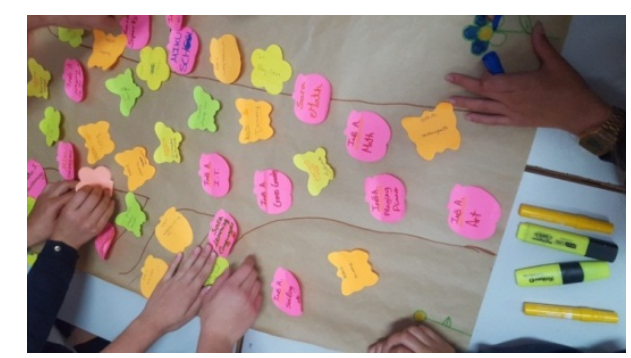

Image 2. The hands show that the creation of the Passion Tree was a real cooperation. The American School of Las Palmas, 2018

Observations: The communication was enriched. They revealed previously unknown details about each other. The self-esteem of every participant was strengthened, and the communications skills were developed week-by-week. In the beginning, they seemed to be very shy. Naming three things that they love doing or are passionate about was not an easy task. It took some weeks for the children to gain routine, and become confident at these conversations. The concept of giving attention to others was better and better understood as time went by. Children received immediate feedback. They were inspired by the care received. The creation of the tree and the flowers involved creativity, arts, and crafts. All the "passion flowers" were collected in individual folders, and a great feedback-book was created from them, witnessing the personal development and passion. 


\subsection{Critical thinking and collaboration}

Critical Thinking and Collaboration are crucial 21st Century skills that should be promoted in school as well. The second Module of S-Cool Days Program focuses on the most exciting part of critical thinking, encourages the children to express gratitude, and say "thank you" to each other.

How do we realize that? In the first module, they learn to identify and express their strengths. In the second module they do the same thing with peers: follow each other's behavior, and say "Thank You" for any help, or any nice message received. Although they have a great routine in giving "Likes" in social media, they start seemingly shy when doing the same thing in the real world, face to face, in the classroom. After the first weeks spent being shy, they got far better, and this part of the Program became the most popular among kids as expressed on the closure meeting.

To create a culture and not a competition, the children collect the "Thank You Coupons" together and spend it on a commonly accepted purpose, such as class excursion, or a bakery visit. The final aim can only be realized if all members collaborate: they start helping each other or start inviting more kids to the basketball play in the break. Otherwise, there is no occasion to thank for. When participating in this part of the Program children started to realize how many beautiful things were and are happening to them in the real world as well. It was interesting to follow how they got more and more conscious about that, started to involve the teachers in the feedback process; some kids even the school. The focus of attention moved from exclusion to inclusion, from hurting each other to helping each other.

This module provides an opportunity to learn monitoring and betterexpressing emotions. The Coupon itself is designed individually, and any creative self-expression is welcomed. The value does not count; the only rule is to name three classmates or teachers, find three activities and say "thank you."

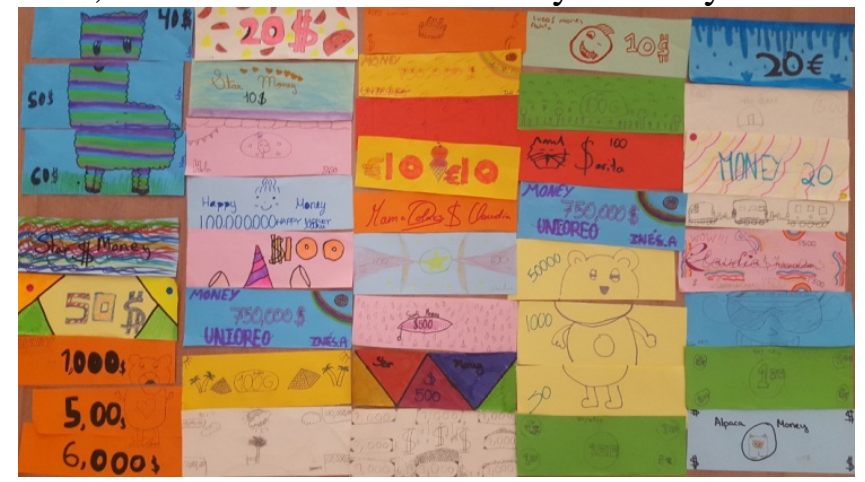

Image 3. Sample - Thank You Coupons, designed by the 6th Graders in the American School of Las Palmas, Spain. The picture shows how creative were the individual "artists". The reason for saying thank you is written on the back side of each "banknote", this way it remains always there, a nice memory from each other. 
To summarize: The S-Cool Days Program introduces new routines that are intended to reframe the current school-practices, and focuses on the development of skills needed in the 21st century. School habits are reframed, new values are introduced, aiming to build a classroom community, which is converted to be the 'comfort zone,' where every child may feel safe and may follow his dreams, find out their highest purpose, and start acting on realizing that. This way a school may not only offer a refreshing alternative to the virtual world of the computer games but can focus on developing new skills, transform learning into a more attractive choice.

Observation: S-Cool Days program might be an opportunity for "bullying prevention" purposes. To show how fragile a school-community might be, let me share follow-up information about one of the participating classes. The Program was finished in June. In the next semester, two boys have arrived to the class, who logically- didn't participate in the S-Cool Days Program. They started to compete from the arrival on, and the "race" ended up in bullying by March. The other boys, who were in the class during S-Cool Days Program, did not contribute to the chase, either to the bullying. On the contrary, one of them raised the attention of the teacher to the issue and asked for help.

\section{Research}

At conferences I often hear the question: It is easy to maintain a virtual community of children of diverse age groups, yet sharing a common interest, but how to build up a thriving community in the real world, in the Aula, with children at the same age and to have a diverse interest. I was curious if S-Cool Days Program may answer this question? Can we reach any change by introducing new routines within the real social network of the class? To measure changes in the classroom network, to reveal the inner dynamics of the groups I designed a straightforward questionnaire, based on the methodology of "sociometric examination" by Ferenc Merei[3]. We asked the kids to share whom they would invite to different occasions, which they would prefer to spend their free time. The same questionnaire was used in all of the three schools, in both countries: in Spain and Hungary.

\section{Results}

\subsection{Social maps of the classes before and after the program}

In each of the three projects, in each school-types, and each age groups there were more mutual common choices after the project than before. In each of the groups, new "common shared choices" were established. However, eight weeks were not 
enough to enclose all of those who have been at the periphery for months or even years.

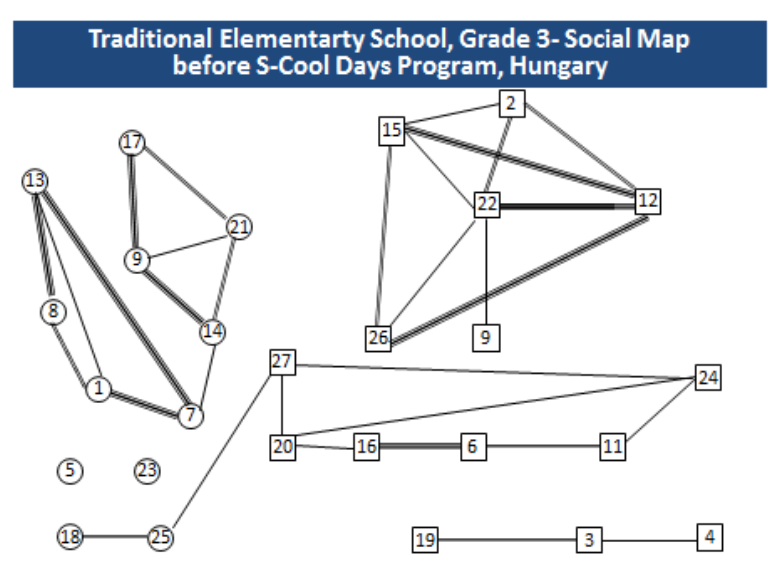

Figure 1. The "before map" shows the network of mutual common choices in the class of Grade 3, Hungary. The circles indicate the girls, square the boys. There were 2 groups of girls, and 3 groups of boys before new routines of the S-Cool Days Program were introduced.

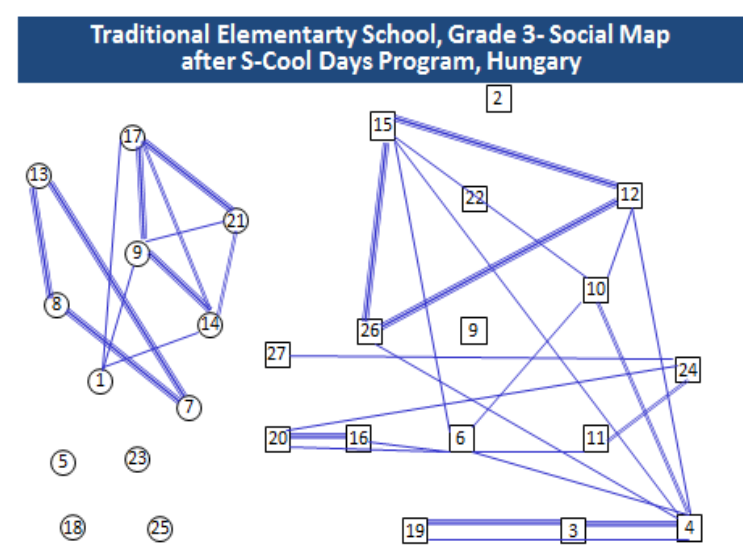

Figure 2. The "after map" shows the mutual common choices in the class, Grade 3, Hungary. The circles indicate the girls, square the boys. The results indicate strong changes in the relationship-network of the boys. The 3 separated groups started to build up mutual connections with each other. The 2 groups of girls started to get closer to each other as well, but the new network is not as strong as at the boys.

The program has delivered similar results in all cities, and all cultures. The new classroom environment welcomes, comforts, and prepares for development any 
child, regardless of the economic or social background, current family status, spoken language, the mother tongue of the participating children, the nationalities present in the classroom.

Table 1. The demographics and other characteristics of the schools where the S-Cool Days Program was introduced

\begin{tabular}{|l|l|c|c|l|l|l|}
\hline Reference Group & School & Grade & Age & Teacher(s) & Classroom Environment & Language \\
\hline $\begin{array}{l}\text { A, American School of } \\
\text { Las Palmas, Spain }\end{array}$ & $\begin{array}{l}\text { Middle } \\
\text { School }\end{array}$ & 6 & 12 & $\begin{array}{l}\text { Rosa Ccabrera, } \\
\text { Mathematics teacher }\end{array}$ & $\begin{array}{l}\text { multicultural environment, } \\
\text { children from Spain, Italy, China } \\
\text { Hungary, Japan, Alaska, US }\end{array}$ & $\begin{array}{l}\text { English, } \\
\text { Spanish }\end{array}$ \\
\hline $\begin{array}{l}\text { B, Budapest School, } \\
\text { Hungary, Innovative } \\
\text { School - Start up, Private }\end{array}$ & Elementary & 1 & 6 & $\begin{array}{l}\text { Sara Porteleki, } \\
\text { Homeroom Teacher }\end{array}$ & children from Hungary only & Hungarian \\
\hline $\begin{array}{l}\text { C, Dunaharaszti state } \\
\text { owned public school, } \\
\text { Hungary }\end{array}$ & Elementary & 3 & 10 & $\begin{array}{l}\text { Katalin Jozan, } \\
\text { Marianna Turzo } \\
\text { Homeroom teachers }\end{array}$ & children from Hungary only & Hungarian \\
\hline
\end{tabular}

\subsection{Results - reflections of participating teachers}

Reference Group A - American School in Spain, Rosa Cabrera, Mathematics teacher: The main benefits the teachers noticed were the following: the group behavior and dynamics improved a lot. The boys and girls integrated. There was an increase of self-awareness and the impact of their behavior in the class. Girls and boys were more integrated, were more open to working in mixed groups when it got to doing projects. Students' emotional intelligence increased after the program. They seemed to care more about each other's hobbies, interests, and feelings. They were more united, more open-minded and understood others' point of view better than before. We think the results are sustainable.

Reference Group B - Sara Porteleki Homeroom teacher. "As a consequence of the S-Cool Days Program, this year is tranquil in our group. I experience, that they take care a lot more towards each-other, give and receive more feedback, to us as well. It makes working very inspiring and motivating for me.

Reference Group C - Katalin Jozan, homeroom teacher: "Socio-economic issues were present in the class before we started the program. One thing I realized was that those performing weaker before began to grow with the others and became better day by day. They began to think more profoundly. They have learned a skill for life. Another benefit, which I experienced was that they started to give positive feedback to us, teachers. It is a very touching feeling.

Reference Group C - Marianna Turzo, homeroom teacher: The most significant difference, I still can't believe in experiencing every day, is the way they, especially the boys started to care about each other. They stopped criticizing each other, understood the concept of giving attention to others, knowing, that they will 
receive it back in a few minutes. The situation in the class was dramatic among boys. They were behaving in a way that detention was always in the air. Now, after the Program, they transformed into emotionally conscious kids. They do not act furiously; on the contrary, they have started to take care of each other, help those who are in need, embrace those who have a bad day. This change is just unbelievable."

\section{Next Steps}

\subsection{Next Steps - School level}

The S-Cool Days Program is currently running in the Budapest School, the School Startup of Peter Halacsy, the successful Start-upper, founder of PREZI; in Budapest, Hungary.

\subsection{Next Steps - International level}

There is a plan to involve more schools into the S-Cool Days Program. More information can be found on the Program home page. [4]

\section{References - Websites}

[1]https://en.wikipedia.org/wiki/21st_century_skills

[2]https://www.mediacom.com/uk/think/reports/connected-kids-2017-report

[3] https://en.wikipedia.org/wiki/Ferenc_M\%C3\%A9rei

[4]www.scooldays.org 